\title{
X-ray emission from the remarkable A-type star HR 8799 (Research Note)
}

\author{
J. Robrade and J. H. M. M. Schmitt
}

Universität Hamburg, Hamburger Sternwarte, Gojenbergsweg 112, 21029 Hamburg, Germany

e-mail: jrobrade@hs . uni-hamburg.de

Received 8 January 2010 / Accepted 31 March 2010

\section{ABSTRACT}

\begin{abstract}
We investigate a Chandra observation of the remarkable planet-bearing A5V star HR 8799, more precisely classified as a $\mathrm{kA} 5 \mathrm{hF} 0 \mathrm{~mA} 5$ star. We search for intrinsic X-ray emission, a diagnostic for studying the possible activity of intermediate-mass stars. In the regime of mid/late A-type stars a strong decline in magnetic activity occurs towards hotter stars because of the vanishing of the outer convection zone. We clearly detect HR 8799 at soft X-ray energies with the ACIS-S detector in a 10 ks exposure; minor $\mathrm{X}$-ray brightness variability is present during the observation. The coronal plasma is described well by a model with a temperature of around $3 \mathrm{MK}$ and an X-ray luminosity of about $L_{\mathrm{X}}=1.3 \times 10^{28} \mathrm{erg} / \mathrm{s}$ in the $0.2-2.0 \mathrm{keV}$ band, corresponding to an activity level of $\log L_{\mathrm{X}} / L_{\mathrm{bol}} \approx-6.2$. Altogether, these findings point to a rather weakly active and given a RASS detection, long-term stable X-ray emitting star. The X-ray emission from HR 8799 resembles those of late A/early F-type stars, in agreement with its classification on the basis of hydrogen lines and its effective temperature determination and thus resolving the apparent discrepancy with the standard picture of magnetic activity that predicts mid A-type stars to be virtually X-ray dark.
\end{abstract}

Key words. stars: activity - stars: coronae - stars: individual: HR 8799 - X-rays: stars

\section{Introduction}

The star HR 8799 (V 342 Peg, HD 218396) is usually classified as spectral type A5 V, located at a distance of $39.9 \mathrm{pc}$, and has a visible magnitude of $V=5.96$ mag with $B-V=0.23$. It is an exceptional and so far unique star, which is $\lambda$ Bootis, $\gamma$ Doradus and Vega-like at the same time. The $\gamma$ Doradus stars are a class of pulsating stars that typically reside at the cool edge of the Cepheid instability strip with spectral types mid-A to midF. The star HR 8799 was linked to the class of $\lambda$ Bootis stars by Gray \& Kaye (1999). The $\lambda$ Bootis stars are chemically peculiar, metal-poor (in particular, for the Fe-peak elements, but with the exception of $\mathrm{C}, \mathrm{N}, \mathrm{O}$ ) A-type stars that do not exhibit ordered large-scale magnetic fields as observed in Ap stars (Bohlender \& Landstreet 1990). Gray \& Kaye (1999) derived a metallicity of $[\mathrm{M} / \mathrm{H}]=-0.47$ for $\mathrm{HR} 8799$ and determined its stellar parameters to be $M=1.47 \pm 0.30 M_{\odot}, R=1.34 \pm 0.05 R_{\odot}$, $L=4.92 \pm 0.41 L_{\odot}\left(1.9 \times 10^{34} \mathrm{erg} / \mathrm{s}\right)$, and $T_{\text {eff }}=7430 \pm 75 \mathrm{~K}$. They assigned it the spectral type $\mathrm{kA} 5 \mathrm{hF} 0 \mathrm{~mA} 5 \mathrm{v} \lambda$ Boo to indicate that HR 8799 is a mild $\lambda$ Bootis star that exhibits the A5 standard in the CaII K line as well as in other metallic lines; however, the hydrogen profiles (and thus effective temperature) are in closer agreement with the F0 standard. The $\lambda$ Bootis phenomenon is thought to be caused by the accretion of metaldepleted material, i.e., external processes, in line with the finding that HR 8799 is also a "Vega-like" star that shows a far-IR excess flux at $60 \mu \mathrm{m}$ from circumstellar dust in IRAS data (Sadakane \& Nishida 1986). In addition, there is strong evidence of a dusty debris disk from IR observations (Zuckerman \& Song 2004).

There has been renewed interest in HR 8799 since the detection of multiple orbiting planets by direct imaging (Marois et al. 2008). The three planets are located at distances of several tens $(24,38,68) \mathrm{AU}$ and have masses around $10 M_{\mathrm{J}}$ each.
The authors derived an age of about $60 \mathrm{Myr}$ (30-150 Myr) from various lines of evidence, thus HR 8799 is probably relatively young. While stellar radiation, especially of high energy such as $\mathrm{UV}$ and X-rays, influences the evolution of circumstellar material and thus planet formation, the planets themselves are most likely not important in the generation of X-ray emission in these stellar systems. Although its $V \sin i=38 \pm 2 \mathrm{~km} \mathrm{~s}^{-1}$ (Kaye \& Strassmeier 1998) is only moderate for an A-type star and the exact orientation of its rotation axis is unknown, an astrometric analysis of the detected planetary system suggests a rather low inclination and consequently a rapidly rotating star, e.g. $V_{\text {rot }} \gtrsim 100(200) \mathrm{km} \mathrm{s}^{-1}$ for $i \lesssim 20(10)^{\circ}$.

Hints of X-ray emission from HR 8799 came from RASS (ROSAT All-Sky-Survey) data by means of a cross-correlation search between X-ray positions and bright A-type stars (Hünsch et al. 1998). They found that the position of the soft X-ray source 1RXS J230729.0+210802 matches quite well with the one of HR 8799; however, the positional uncertainty and low number of detected counts (10 photons) prevent a detailed investigation of HR 8799 with the ROSAT data. On the other hand, Kaye \& Strassmeier (1998) attributed the chromospheric Ca II K flux of HR 8799 to be of basal origin. From the X-ray point of view, HR 8799 is remarkable because an A5 star is expected to be virtually X-ray dark in the standard paradigm that is applicable to main sequence stars. In this scheme, X-ray emission is expected from magnetic activity in the regime of "cool stars" (spectral type around A7 and later) and from wind-shocks in the regime of "hot stars" (B2 and earlier); intermediate spectral types are virtually X-ray dark. Exceptions are found among young Herbig AeBe stars and peculiar Ap/Bp stars, where fossil magnetic fields are thought to play a major role in the generation of X-rays. This picture is well established by X-ray observations with Einstein and ROSAT, showing that stellar activity develops 

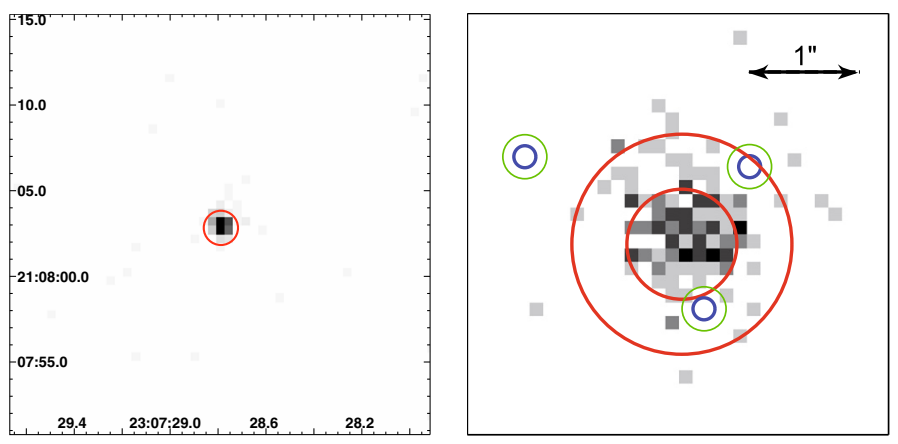

Fig. 1. Left: ACIS-S image of HR 8799 and optical position. Right: subpixel image with the three planets marked (see text for details).

in late A-type stars and increases strongly towards cooler stars, with activity levels being in the range of $\log L_{\mathrm{X}} / L_{\mathrm{bol}}=-3, \ldots,-7$ (e.g. Schmitt et al. 1985; Schmitt 1997). An XMM-Newton observation of the fast rotating A7 star Altair confirmed the presence of weak magnetic activity and coronal X-ray emission (Robrade \& Schmitt 2009). The vanishing of magnetic activity in mid A-type stars is expected theoretically and confirmed by other activity indicators; for instance FUSE observations of main sequence stars have shown the disappearance of chromospheric emission lines at effective temperatures above $T_{\text {eff }} \approx 8200 \mathrm{~K}$ (Simon et al. 2002).

In this paper, we present the results of our analysis of a Chandra ACIS-S observation of HR 8799. Our paper is structured as follows: in Sect. 2, we describe the observation and data analysis, in Sect. 3, we present our results and summarize our findings in Sect. 4.

\section{Observations and data analysis}

The target HR 8799 was observed by Chandra with the backilluminated S3 chip of the ACIS-S detector in August 2009 for about $10 \mathrm{ks}$ (Obs.-ID 10975). We used the standard software package CIAO 4.1 to analyze the data, including the tool "wavedetect" to derive the source position. Source photons were found in the $0.15-2.0 \mathrm{keV}$ energy range, where we detected 137 photons in a $2^{\prime \prime}$ circular region around the position of HR 8799 with an expected background of 0.2 photons as deduced from nearby source-free regions. These photons are denoted as source photons in the following and enable a more detailed study of the X-ray properties of HR 8799, compared to the ROSAT data.

Our spectral analysis was performed using XSPEC V12.3 (Arnaud 1996) and we required a minimum of 15 counts per spectral bin in our modeling. To model the spectra, we used photo-absorbed, multi-temperature APEC models; all abundances are relative to solar values as given by Grevesse \& Sauval (1998). We find that no absorption component is required to describe the obtained X-ray spectrum and that fixing interstellar absorption to a level of $N_{\mathrm{H}}=10^{19}-10^{20} \mathrm{~cm}^{-2}$ does not significantly alter the results.

\section{Results}

\subsection{Images and light curves}

In the left panel of Fig. 1 we show the obtained X-ray image of the source and the optical position of HR 8799 , denoted by a circle with a $1^{\prime \prime}$ radius, corresponding to $\approx 95 \%$ encircled X-ray energy. The right panel shows a zoomed image of the source,

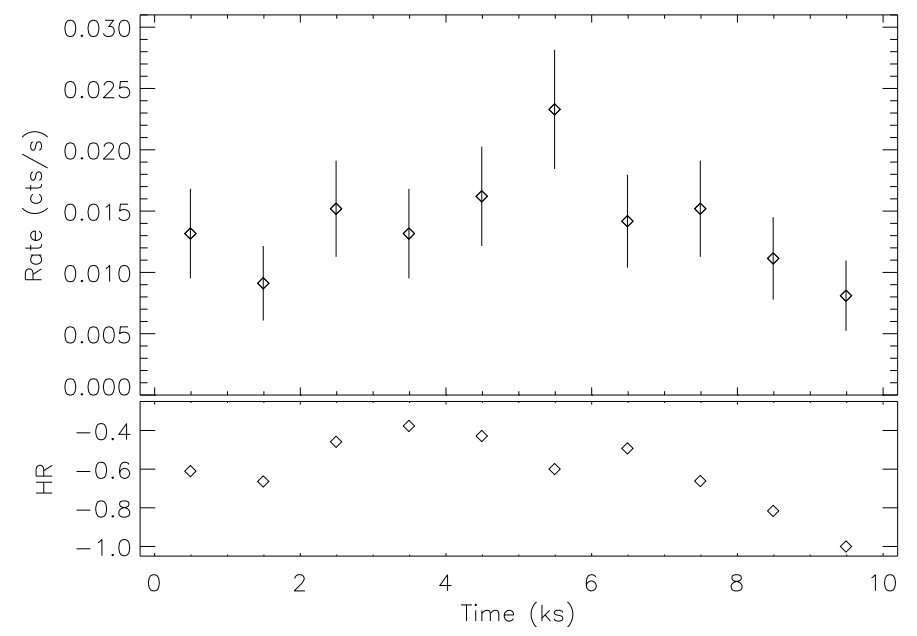

Fig. 2. Light curve and hardness ratio of HR 8799 (1 ks binning).

the outer circle being the same as in the left panel, the inner half-sized circle corresponds to $65 \%$ encircled energy. In addition, the planetary positions are marked (small circles with $0.1^{\prime \prime}$ and $0.2^{\prime \prime}$ radius). Only one X-ray source is present, it appears point-source like and its position agrees with an absolute offset of only $0.2^{\prime \prime}$ very well with the optical position of the A-type star HR 8799. No significant excess emission is found at any of the positions of the detected planets (separations to HR 8799 between $0.6^{\prime \prime}$ and $1.7^{\prime \prime}$ ) or at the positions of the substellar companions that were detected as point-source candidates in a coronographic survey performed with NICMOS/HST at distances of 13.7" and 15.7" (Lowrance et al. 2005). Possible $\mathrm{X}$-ray emission from any of these objects is at least two orders of magnitude fainter than those from HR 8799. HR 8799 is thought to be a single star and given the accurate source position obtained from the ACIS data, we attribute the detected X-ray emission exclusively to the A5 star HR 8799.

Bohlender \& Landstreet (1990) placed upper limits of a few hundred Gauss on the longitudinal magnetic field component in a sample of $\lambda$ Bootis stars, not including HR 8799 and excluding the existence of strong, ordered fields. However, small-scale magnetic fields as expected for a corona that originates from magnetic activity may easily be present. The X-ray emission from HR 8799 implies that the star has a magnetic field, thus $\lambda$ Bootis stars are not exclusively non-magnetic.

To investigate the X-ray variability of HR 8799 , we produced a light curve with $1 \mathrm{ks}$ binning from the source photons (Fig. 2, upper panel). The light curve is moderately variable; the count rate changes by up to a factor of about two, although the precise level of the variability is not well constrained. We also studied light curves with shorter time bins of e.g. $100 \mathrm{~s}$, but found no indications of strong short-term variability that would be present for burst-like flares.

We studied the origin of the observed variability and searched for spectral variations related to the changes in X-ray brightness by determining the respective hardness ratio $H R=$ $(H-S) /(H+S)$ for each time bin, with $S=0.15-0.7 \mathrm{keV}$ and $H=0.7-2.0 \mathrm{keV}$ being the respective photon energy bands (see lower panel, Fig. 2). Spectral lines are unresolved in the ACIS spectrum; however, the emission below $0.7 \mathrm{keV}$ is dominated by cooler plasma with line peak formation temperatures in the range of $1-4 \mathrm{MK}$, while above $0.7 \mathrm{keV}$ lines from hotter plasma ( $25 \mathrm{MK}$ ) dominate. If enhanced magnetic activity were the origin of the X-ray brightening, one would expect a correlation of 
J. Robrade and J. H. M. M. Schmitt: X-ray emission from the remarkable A-type star HR 8799 (RN)

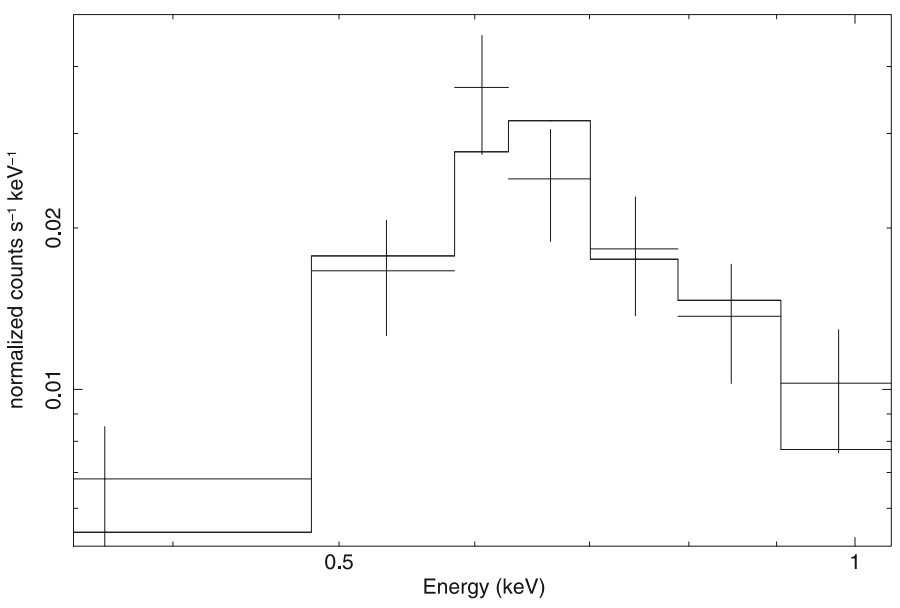

Fig. 3. ACIS-S spectrum of HR 8799 with applied spectral model.

the X-ray brightness with the hardness of the emission. The error in the individual hardness ratio bins is about \pm 0.25 , but indeed harder emission is observed during X-ray brighter phases. A Spearman's rank correlation gives a chance probability of 0.07 that the correlation is caused by a statistical fluctuation for the ten time bins used. Thus, variable magnetic activity is the likely cause of the observed moderate X-ray brightness variability, whereas more extreme flaring events such as those observed on active stars, are not present on HR 8799 during our observation.

\subsection{Spectral analysis}

We determined the global spectral properties of HR 8799 by fitting the ACIS spectrum with multi-temperature spectral models. The metallicity of the coronal plasma can only be poorly constrained with the existing data and was kept fixed. Using the photospheric value of HR 8799, determined to be 0.33 solar abundances (Gray \& Kaye 1999), already a one-temperature model was found to be sufficient to describe the data. The corona therefore also seems to exhibit the photospheric sub-solar composition. Adopting solar abundances results in correspondingly lower emission measure, albeit the quality of the fit is slightly poorer for a one-temperature model. The corona therefore is more accurately described by the photospheric sub-solar metallicity, but here the indications from the X-ray data are sparse. We note that a similarly good fit can be obtained with solar metallicity and two, however poorly constrained, temperature components. We show the X-ray spectrum and the best-fit onetemperature model in Fig. 3, the derived spectral properties are summarized in Table 1.

From the best fit model, we obtain an X-ray luminosity of $L_{\mathrm{X}}=1.3 \times 10^{28} \mathrm{erg} / \mathrm{s}$ in the $0.2-2.0 \mathrm{keV}$ band, corresponding to a surface flux of $\log F_{X}=5.1 \mathrm{erg} \mathrm{cm}^{-2} \mathrm{~s}^{-1}$ and an activity level of $\log L_{\mathrm{X}} / L_{\mathrm{bol}}=-6.16$, indicating that HR 8799 is despite its youth a rather weakly active star. Since HR 8799 is about an order of magnitude X-ray brighter than the average Sun, the X-ray flux at the position of the closest of the detected planets corresponds to the solar X-ray flux that is received by Saturn. To compare our result with the RASS measurement, we also determine the X-ray luminosity for the ROSAT $0.1-2.4 \mathrm{keV}$ band from the spectral model, leading to a value of $L_{X}=1.6 \times 10^{28} \mathrm{erg} / \mathrm{s}$. The ROSAT X-ray luminosity of $L_{\mathrm{X}}=1.5 \times 10^{28} \mathrm{erg} / \mathrm{s}$, obtained from a hardness-dependent energy conversion factor, is basically the
Table 1. Spectral fit results derived from ACIS-S data.

\begin{tabular}{lrrl}
\hline \hline Par. & \multicolumn{2}{c}{ 1-T models } & Unit \\
\hline T1 & $0.26 \pm 0.03$ & $0.26 \pm 0.03$ & $\mathrm{keV}$ \\
Abund. & 0.33 & 1.0 & solar \\
EM1 & $1.9 \pm 0.3 \times 10^{51}$ & $6.9 \pm 1.1 \times 10^{50}$ & $\mathrm{~cm}^{-3}$ \\
\hline$\chi_{\text {red }}^{2}$ (d.o.f.) & $0.83(5)$ & $1.14(5)$ & \\
\hline
\end{tabular}

same as that obtained with Chandra more than 25 years later, which is indicative of long-term stable X-ray emission.

\subsection{HR 8799 in the context of A-type stars}

The activity level as well as the X-ray luminosity of HR 8799 are about an order of magnitude higher than those of magnetically active mid to late A-type stars such as Alderamin ( $\alpha$ Cep) or Altair ( $\alpha$ Aql), which are both of spectral type A7. These stars have X-ray luminosities of one to a few times $10^{27} \mathrm{erg} / \mathrm{s}$ and activity levels of $\log L_{\mathrm{X}} / L_{\text {bol }} \lesssim-7$. Compared to Altair, the hottest magnetically active star studied in detail at X-ray energies (Robrade \& Schmitt 2009), the X-ray surface flux of HR 8799 is higher by about a factor of 20; further the average coronal temperature of HR 8799 is with $3.0 \mathrm{MK}$ slightly higher than that of Altair which is about $2.5 \mathrm{MK}$, but within the errors this difference is not very significant. This matches the finding that Altair's activity level is at $\log L_{\mathrm{X}} / L_{\mathrm{bol}}=-7.4$ very low, even when compared to the weakly active stars HR 8799 . However, the ultra-fast rotator Altair is already close to its maximum possible activity level and a significant spin-up would disrupt the star. Consequently, the dynamo mechanism in HR 8799 must therefore be more efficient than that in Altair. The efficiency of a solar-type dynamo is proportional to the inverse square of the Rossby-number $\left(\operatorname{Ro}=P / \tau_{\mathrm{c}}\right)$, where $P$ denotes the rotational period and $\tau_{\mathrm{c}}$ the convective turnover time. A significantly shorter rotation period for HR 8799 is ruled out by considering stellar dimensions, $V \sin i$ and geometry; thus, a deeper convection zone would provide a natural explanation for the required efficiency.

This is in line with the finding that for a spectral classification as an A5 star, the determined $T_{\text {eff }} \approx 7400 \mathrm{~K}$ from Gray $\&$ Kaye (1999) is rather low. Thus one might assume that its internal structure is more accurately described by a F0 star. In this case, the outer convection zone would easily provide sufficient dynamo action to generate the observed X-ray emission from magnetic activity. The still rather low activity level of $\log L_{\mathrm{X}} / L_{\text {bol }} \approx-6.1$ (active late-type stars have $\log L_{\mathrm{X}} / L_{\mathrm{bol}} \approx$ $-3)$ that is required to generate the observed X-ray emission from HR 8799 is easily achieved in this case. Furthermore, as is also the case for Altair, the surface of HR 8799 is not necessarily homogeneous and specific surface areas such as an equatorial bulge may contribute predominantly to the X-ray emission.

The activity level and the coronal properties support the hypothesis that the often used "metallic" classification as A5 star does not reflect the properties that are related to the magnetic activity phenomena. From the X-ray point of view, HR 8799 resembles more closely a late A or early F-type star. This is in line with its classification based on hydrogen lines and its effective temperature derived from spectral modelling. The X-ray emission from HR 8799 therefore appears rather natural. Sufficiently sensitive searches with other indicators should also reveal signs of magnetic activity phenomena on this remarkable star. 


\section{Summary and conclusions}

From a $10 \mathrm{ks}$ Chandra observation of HR 8799, we derive the following results and conclusions.

1. We have detected soft X-ray emission from the extraordinary A5/F0 V star HR 8799. The derived X-ray luminosity of about $L_{\mathrm{X}}=1.3 \times 10^{28} \mathrm{erg} / \mathrm{s}$ in the $0.2-2.0 \mathrm{keV}$ band corresponds to an activity level of $\log L_{\mathrm{X}} / L_{\mathrm{bol}} \approx-6.2$, which is indicative of a weakly active star.

2. The ACIS data confirms the ROSAT detection, significantly improves the positional accuracy and additionally enables a deeper study of the X-ray properties of HR 8799. Its X-ray emitting coronal plasma has an average temperature of about 3.0 MK, typical of a star with a rather low activity level. Minor X-ray brightness variations are present in our observation; the accompanying spectral changes indicate variable magnetic activity.

3. Overall, the X-ray properties of HR 8799 resemble those of mildly active late $\mathrm{A} /$ early $\mathrm{F}$ stars, rather than those of mid/late A-type stars. Keeping in mind that the attributed spectral type for HR 8799 depends on the classification criterion, the one based on hydrogen lines (F0) is most consistent with its X-ray properties.

Acknowledgements. This work is based on observations obtained with Chandra. J.R. acknowledges support from DLR under 50QR0803.

\section{References}

Arnaud, K. A. 1996, in Astronomical Data Analysis Software and Systems V, ed. G. H. Jacoby, \& J. Barnes, ASP Conf. Ser., 101, 17 Bohlender, D. A., \& Landstreet, J. D. 1990, MNRAS, 247, 606 Gray, R. O., \& Kaye, A. B. 1999, AJ, 118, 2993

Grevesse, N., \& Sauval, A. J. 1998, Space Sci. Rev., 85, 161 Hünsch, M., Schmitt, J. H. M. M., \& Voges, W. 1998, A\&AS, 132, 155 Kaye, A. B., \& Strassmeier, K. G. 1998, MNRAS, 294, L35

Lowrance, P. J., Becklin, E. E., Schneider, G., et al. 2005, AJ, 130, 1845 Marois, C., Macintosh, B., Barman, T., et al. 2008, Science, 322, 1348 Robrade, J., \& Schmitt, J. H. M. M. 2009, A\&A, 497, 511

Sadakane, K., \& Nishida, M. 1986, PASP, 98, 685

Schmitt, J. H. M. M. 1997, A\&A, 318, 215

Schmitt, J. H. M. M., Golub, L., Harnden, Jr., F. R., et al. 1985, ApJ, 290, 307 Simon, T., Ayres, T. R., Redfield, S., \& Linsky, J. L. 2002, ApJ, 579, 800

Zuckerman, B., \& Song, I. 2004, ApJ, 603, 738 